OPEN ACCESS

Edited by:

Lihua Xiao,

South China Agricultural University,

China

Reviewed by:

Aiqin Liu,

Harbin Medical University, China

Guangneng Peng,

Sichuan Agricultural University, China

Yu Juan Shen,

National Institute of Parasitic

Diseases, China

*Correspondence:

Meng Qi

qimengdz@163.com

Longxian Zhang

zhanglx8999@henau.edu.cn

Specialty section:

This article was submitted to

Infectious Diseases,

a section of the journal

Frontiers in Microbiology

Received: 22 May 2019 Accepted: 05 August 2019

Published: 16 August 2019

Citation:

Zhao A, Li D, Wei Z, Zhang Y,

Peng Y, Zhu Y, Qi M and Zhang L

(2019) Molecular Detection and Genotyping of Enterocytozoon

bieneusi in Racehorses in China.

Front. Microbiol. 10:1920.

doi: 10.3389/fmicb.2019.01920

\section{Molecular Detection and Genotyping of Enterocytozoon bieneusi in Racehorses in China}

\author{
Aiyun Zhao ${ }^{1}$, Dongfang Li $^{2}$, Zilin Wei ${ }^{1}$, Ying Zhang ${ }^{1}$, Yushi Peng ${ }^{3}$, Yixuan Zhu' ${ }^{1}$, Meng Qi ${ }^{\text {* }}$ \\ and Longxian Zhang ${ }^{2 *}$ \\ ${ }^{1}$ College of Animal Science, Tarim University, Alar, China, ${ }^{2}$ College of Animal Science and Veterinary Medicine, Henan \\ Agricultural University, Zhengzhou, China, ${ }^{3}$ Equivets, Beijing, China
}

Enterocytozoon bieneusi is a widely distributed human and animal pathogen. However, few data are available on the distribution of E. bieneusi genotypes in racehorses. In this study, $621 \mathrm{fecal}$ specimens were collected from racehorses at 17 equestrian clubs in 15 Chinese cities. E. bieneusi was detected via nested polymerase chain reaction (PCR) amplification of the internal transcribed spacer (ITS) gene. The overall infection rate of E. bieneusi was 4.8\% (30/621). Statistically significant differences were found in the prevalence of this parasite among the equestrian clubs $\left(\chi^{2}=78.464, d f=16\right.$, $p<0.01)$ and age groups $\left(x^{2}=23.686, d f=1, p<0.01\right)$, but no sex bias was found among the racehorses for the $E$. bieneusi infections $\left(\chi^{2}=1.407, d f=2, p>0.05\right)$. Ten E. bieneusi genotypes were identified, including seven known genotypes (EbpC, EbpA, Peru6, horse1, horse2, CAF1, and TypelV) and three novel genotypes $(\mathrm{HBH}-1, \mathrm{SXH}-$ 1, and BJH-1). Phylogenetic analysis showed that EbpC, EbpA, Peru6, horse2, CAF1, TypelV, BJH-1, and $\mathrm{SXH}-1$ belonged to Group 1 of $E$. bieneusi, $\mathrm{HBH}-1$ belonged to Group 2, and horse2 belonged to Group 6. Our findings advance the current knowledge of $E$. bieneusi prevalence and genotypes in racehorses in China.

Keywords: Enterocytozoon bieneusi, racehorse, prevalence, genotype, zoonotic

\section{INTRODUCTION}

Enterocytozoon bieneusi, an obligate intracellular eukaryotic pathogen, infects a wide variety of vertebrates and invertebrates, including humans (Santín and Fayer, 2011). Clinical symptoms caused by E. bieneusi vary depending on the health status of the infected hosts. Asymptomatic infections or self-limiting diarrhea often occur in immunocompetent or healthy individuals, while chronic or life-threatening diarrhea occur in immunocompromised individuals (Didier and Weiss, 2006; Maikai et al., 2012). Most E. bieneusi infections in humans result from fecal-oral transmission of spores from infected hosts through contaminated food or water. A foodborne outbreak was reported in Sweden in 2009 (Decraene et al., 2012). Environmentally resistant, infective spores have been detected in various water bodies, including irrigation water, a drinking-source watershed, recreational water, and wastewater from treatment plants, suggesting the possibility of waterborne transmission (Ben et al., 2012; Li et al., 2012; Galván et al., 2013). Because of the clinical and public health importance of E. bieneusi, the National Institutes of Health has ranked it on the category 
B list ${ }^{1}$, and the Environmental Protection Agency has placed it on the microbial contaminant candidate list of concern for waterborne transmission (Didier et al., 2009). To date, based on sequence analysis of the ribosomal internal transcribed spacer (ITS) gene, 474 E. bieneusi ITS genotypes have been identified, and 11 phylogenetic groups have been recognized (Li et al., 2019). Group 2 does not include ruminant-specific genotypes, nor does it specify the zoonotic potential of some genotypes (notably BEB4, BEB6, I, and J); thus, Group 2 genotypes are not host-specific (Wang et al., 2018; Li and Xiao, 2019).

Most reports on E. bieneusi infections in animals involve cattle, sheep and other livestock, pets, non-human primates and wildlife; however, few reports are available on horses (Thellier and Breton, 2008; Santín et al., 2018; Zhang et al., 2018). In 2010, Santín et al. first reported on E. bieneusi infections in horses in Colombia, after which, E. bieneusi infections in horses were reported in Algeria, the Czechia, the United States and China (Table 1). To date, 37 E. bieneusi genotypes have been identified in horses. To our knowledge, the following eight genotypes have been reported as having infected humans: BEB6, CZ3, CS-4, D, EbpA, EbpC, O, and Peru8 (Leelayoova et al., 2006; Thellier and Breton, 2008; Sak et al., 2011; Wang et al., 2013, 2018; Liu et al., 2017; Table 1).

Horses are common animals worldwide and have been used for leisure activities, sports, and working purposes, including agricultural production, transportation, and military combat. In recent years, horse racing, as one of the oldest known sports, has become more popular in China, and the value of racehorses has increased. However, only one study has been published on $E$. bieneusi infections in racehorses in China, reporting the prevalence of this pathogen in two equestrian clubs in Sichuan at $10.4 \%(5 / 48)$ and $9.6 \%$ (5/52; Deng et al., 2016b). To better

${ }^{1}$ https://www.niaid.nih.gov/research/emerging-infectious-diseases-pathogens understand E. bieneusi transmission in racehorses in China, we investigated the occurrence of E. bieneusi genotypes in racehorses from 17 equestrian clubs.

\section{MATERIALS AND METHODS}

\section{Fecal Specimen Collection and DNA Extraction}

From December 2016 to May 2018, 621 fresh fecal specimens were collected from 30 to $50 \%$ of the racehorses at 17 equestrian clubs in 15 Chinese cities (Figure 1 and Table 2). Among the 621 specimens, 74 were from young horses, 547 were from adult horses, 186 were from stallions, 350 were from mares, and 85 were from castrated horses. All horses appeared healthy. Each specimen (30-50 g) was collected directly from the rectum or from the ground immediately after defecation using a sterile disposable latex glove and placed into an individual plastic zip-lock bag. Each individual was identified by the name or number provided by the veterinarians at the equestrian clubs. Specimens were stored in a cooler with ice packs and immediately transferred to the laboratory for testing. The specimens were stored at $4^{\circ} \mathrm{C}$, and DNA was extracted within 1 week after collection.

Ten grams of each fecal specimen was thoroughly mixed with $30 \mathrm{~mL}$ of distilled water. The suspension was passed through a $250-\mu \mathrm{m}$ pore wire mesh sieve and centrifuged at $3000 \times \mathrm{g}$ for $5 \mathrm{~min}$. The precipitates were used for DNA extraction. Genomic DNA was directly extracted from each $(200 \mathrm{mg})$ precipitate using the E.Z.N.A. ${ }^{\circledR}$ Stool DNA Kit (D4015-02, Omega Biotek, Inc., Norcross, GA, United States) per the manufacturer's instructions with minor modifications. The extracted DNA specimens were stored at $-20^{\circ} \mathrm{C}$ prior to polymerase chain reaction $(\mathrm{PCR})$ analysis.

TABLE 1 | Enterocytozoon bieneusi occurrence and genotype distribution among horses worldwide.

\begin{tabular}{|c|c|c|c|}
\hline Country (region) & $\begin{array}{l}\text { No. Positive/No. } \\
\text { examined (\%) }\end{array}$ & Genotype (n) & References \\
\hline Algeria & $15 / 219(6.8)^{\mathrm{a}}$ & CZ3 (2), D (1), horse1 (6), horse2 (1) & Laatamna et al., 2015 \\
\hline $\begin{array}{l}\text { China (Sichuan and } \\
\text { Yunnan) }\end{array}$ & 75/333 (22.5) & $\begin{array}{l}\text { D (1), horse1 (13), horse2 (39), SCO2 (16), SCH1 (1), SCH2 (1), SCH3 (1), SCH4 (1), } \\
\text { YNH1 (1), YNH2 (1) }\end{array}$ & Deng et al., 2016b \\
\hline Czechia & $66 / 377(17.5)$ & $\begin{array}{l}\text { D (34), EbpA (2), G (3), horse1 (7), horse2 (8), horse3 (2), horse4 (1), horse5 (1), } \\
\text { horse6 (1), horse7 (1), horse8 (1), horse9 (1), horse10 (1), horse11 (2), WL15(1) }\end{array}$ & Wagnerová et al., 2012 \\
\hline Switzerland & 0/24 (0) & & Breitenmoser et al., 1999 \\
\hline Spain & 0/10 (0) & & Lores et al., 2002 \\
\hline United States & $7 / 84(8.3)$ & horse1 (7) & Wagnerová et al., 2016 \\
\hline
\end{tabular}

aUnsuccessful resequencing of five positive specimens. Genotypes detected in humans are shown in bold. 


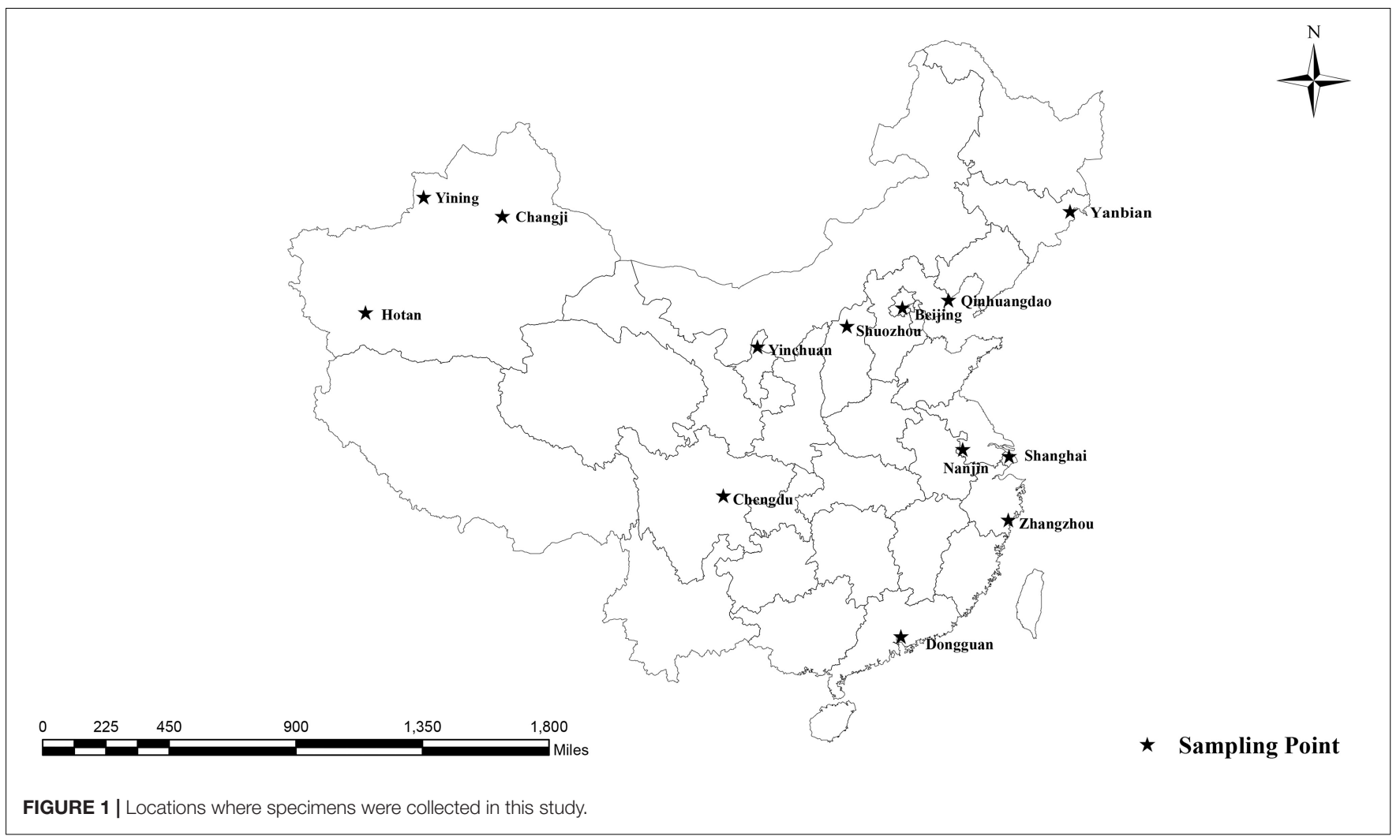

\section{PCR Amplification and Sequence Analysis}

Enterocytozoon bieneusi was detected via nested PCR amplification of the ITS region of the rRNA gene, using the primers and PCR conditions described previously by Buckholt et al. (2002). First, $12.5 \mu \mathrm{L} 2 \times$ EasyTaq PCR SuperMix (TransGene Biotech Co., Beijing, China) was used to amplify each specimen in a $25-\mu \mathrm{L}$ reaction volume containing $10.9 \mu \mathrm{L}$ deionized water, $0.3 \mu \mathrm{M}$ of each primer, $1 \mu \mathrm{L}$ genomic DNA for the primary PCR, and $1 \mu \mathrm{L}$ primary amplification product for the secondary PCR. A positive control (DNA from dairy cattle-derived genotype I) and a negative control (distilled water) were used in each PCR run. PCR amplification was repeated twice on each specimen.

All positive secondary PCR amplicons ( $\sim 390$ bp each) were sent for bidirectional sequencing at GENEWIZ (Suzhou, China). The resultant sequences were assembled using Chromas Pro, version $2.18^{2}$ and compared with the reference sequences in the National Center for Biotechnology Information ${ }^{3}$ database using ClustalX, version $2.1^{4}$ to determine the E. bieneusi genotypes. The nucleotide sequences obtained in this study were submitted to GenBank ${ }^{5}$ under accession numbers MK789437-MK789446.

\footnotetext{
${ }^{2}$ http://technelysium.com.au

${ }^{3}$ https://www.ncbi.nlm.nih.gov/

${ }^{4}$ http://clustal.org/

${ }^{5}$ https://www.ncbi.nlm.nih.gov/genbank/
}

\section{Phylogenetic and Statistical Analyses}

The sequences of the ITS regions of the E. bieneusi genotypes obtained in this study were compared with those previously identified in humans, other animals and the environment. Bayesian inference (BI) and Monte Carlo Markov chain methods were used to construct phylogenetic trees in MrBayes, version 3.2.6 ${ }^{6}$. The general time reversible model $(\mathrm{GTR}+\mathrm{G})$ was the best-fit nucleotide substitution model determined by ModelTest, version $3.7^{7}$. The number of substitutions was set at six, with a proportion of invariable sites. Posterior probability values were calculated by running 1,000,000 generations with four simultaneous tree-building chains. Trees were saved every 1000th generation. At the end of each run, the standard deviation of the split frequencies was $<0.01$, and the potential scale reduction factor approached one. A 50\% majority rule consensus tree was constructed for each analysis using the final $75 \%$ of the trees generated via BI. Analyses were run three times to ensure convergence and insensitivity to prior runs. The maximum clade credibility tree generated by these analyses was viewed and edited using FigTree version 1.3.1 software ${ }^{8}$.

The Statistical Package for the Social Sciences (SPSS, version 22.0, available at https://www.ibm.com) was used for the statistical analyses, including Fisher's exact test and 95\% confidence intervals. All results were considered statistically significant at $p<0.05$.

\footnotetext{
${ }^{6} \mathrm{http}: / /$ mrbayes.sourceforge.net/

${ }^{7}$ http://www.softpedia.com/get/Science-CAD/Modeltest.shtml

${ }^{8}$ https://www.softpedia.com/get/Science-CAD/FigTree-AR.shtml
} 
TABLE 2 | Enterocytozoon bieneusi occurrence and genotype profiles in racehorses in China.

\begin{tabular}{|c|c|c|c|c|c|}
\hline Cities & $\begin{array}{c}\text { No. } \\
\text { Positive/No. } \\
\text { samples (\%) }\end{array}$ & $95 \% \mathrm{Cl}$ & $x^{2}$ & $\begin{array}{c}P \text { - } \\
\text { value }\end{array}$ & $\begin{array}{l}\text { E. bieneusi } \\
\text { genotype (n) }\end{array}$ \\
\hline Beijing1 & 2/32 (6.3) & $0-15.1$ & & & Peru6 (2) \\
\hline Beijing2 & 0/39 & & & & \\
\hline Beijing3 & $3 / 45(6.7)$ & $0-14.2$ & & & EbpC (2), BJH-1 (1) \\
\hline Baicheng & $0 / 29$ & & & & \\
\hline Changji & $9 / 34(26.5)$ & $10.8-42.1$ & & & horse1 (9) \\
\hline Chengdu & $1 / 34(2.9)$ & $0-8.9$ & & & horse1 (1) \\
\hline Dongguan & $1 / 19(5.3)$ & $0-16.3$ & & & EbpA (1) \\
\hline Hotan & $0 / 21$ & & & & \\
\hline Nanjing & $0 / 9$ & 0 & & & \\
\hline Qinhuangdao & $1 / 136(0.7)$ & $0-2.2$ & & & $\mathrm{HBH}-1$ (1) \\
\hline Shanghai & $2 / 84(2.4)$ & $0-5.7$ & & & horse1 (2) \\
\hline Shuozhou & $4 / 15(26.7)$ & $1.3-52.0$ & & & horse1 (3), SXH-1 (1) \\
\hline Wenzhou & $0 / 23$ & & & & \\
\hline Wuhan & $3 / 20(15.0)$ & $0-32.1$ & & & Peru6 (2), horse2 (1) \\
\hline Yanbian & $0 / 14$ & & & & \\
\hline Yinchuan & $0 / 44$ & & & & \\
\hline Zhaosu & $4 / 23(17.4)$ & $0.6-43.2$ & & & $\begin{array}{l}\text { horse1 (1), horse2 (1), } \\
\text { TypelV (1), CAF1 (1) }\end{array}$ \\
\hline Total & $30 / 621(4.8)$ & $3.1-6.5$ & 78.464 & $<0.01$ & $\begin{array}{l}\text { horse1 (16), } \\
\text { Peru6 (4), EbpC (2), } \\
\text { horse2 (2), EbpA (1), } \\
\text { CAF1 (1), TypelV (1), } \\
\text { BJH-1 (1), HBH-1 (1), } \\
\text { SXH-1 (1) }\end{array}$ \\
\hline
\end{tabular}

Genotypes detected in humans are shown in bold.

\section{RESULTS AND DISCUSSION}

Among the 621 racehorse fecal specimens analyzed, 30 (4.8\%) were positive for E. bieneusi, with E. bieneusi detected in 10 equestrian clubs $(58.8 \%)$ from nine cities $(60.0 \%)$. E. bieneusi infection rates were related to the collection sites $\left(\chi^{2}=78.464\right.$, $d f=16, p<0.01$; Table 2); the highest infection rates occurred in equestrian clubs in Shuozhou $(26.7 \%, 4 / 15)$, Changji $(26.5 \%$, 9/34), Zhaosu $(17.4 \%, 4 / 23)$ and Wuhan $(15.0 \%, 3 / 20)$, with the other sampled equestrian clubs having lower infection rates. Globally, the E. bieneusi infection rates in horses range from $0-30.9 \%$. Currently, two studies have reported E. bieneusi infections in horses in China: 30.9\% (81/262) in Xinjiang (Qi et al., 2016) and 22.5\% (75/333) in Sichuan and Yunnan (Deng et al., 2016b), which were higher than those in the racehorses in the present study. This discrepancy may be related to the different management systems used for the horses. Wagnerová et al. (2012) reported that the E. bieneusi infection rate in stabled horses $(26.6 \%, 25 / 94)$ was higher than that in pastured horses $(18.9 \%, 23 / 122)$ and paddocked horses $(11.2 \%, 18 / 161)$ in the Czechia. Deng et al. (2016a) reported E. bieneusi infection rates in China of 29.1\% (48/165) in pastured horses, $26.8 \%(15 / 56)$ in agricultural horses, $10.4 \%$ $(5 / 48)$ in racehorses, and $9.6 \%(5 / 52)$ in equestrian clubs. Racehorses are selected according to their health, are well cared for, and live in good conditions, which may explain
TABLE 3 | Enterocytozoon bieneusi occurrence and genotypes among racehorses of different ages and sex.

\begin{tabular}{|c|c|c|c|c|c|}
\hline $\begin{array}{l}\text { Ages } \\
\text { and sex }\end{array}$ & $\begin{array}{c}\text { No. } \\
\text { Positive/No. } \\
\text { specimens } \\
(\%)\end{array}$ & $95 \% \mathrm{Cl}$ & $x^{2}$ & $\begin{array}{c}P \text { - } \\
\text { value }\end{array}$ & $\begin{array}{l}\text { E. bieneusi } \\
\text { genotype (n) }\end{array}$ \\
\hline Youths & 12/74 (16.2) & $7.6-24.8$ & 23.686 & $<0.01$ & $\begin{array}{l}\text { horse1 (9), horse2 (1), } \\
\text { TypelV (1), CAF1 (1) }\end{array}$ \\
\hline Adults & 18/547 (3.3) & $1.8-4.8$ & & & $\begin{array}{l}\text { EbpA (1), EbpC (2), } \\
\text { horse1 (7), horse2 (1), } \\
\text { Peru6 (4), BJH-1 (1), } \\
\mathrm{HBH}-1 \text { (1), SXH-1 (1) }\end{array}$ \\
\hline Stallion & 9/186 (4.8) & $1.7-8.0$ & 1.407 & $>0.05$ & $\begin{array}{l}\text { horse1 (2), horse2 (2), } \\
\text { CAF1 (1), Peru6 (1), } \\
\text { TypelV (1), HBH-1 (1), } \\
\text { BJH-1 (1) }\end{array}$ \\
\hline Mare & $19 / 350(5.4)$ & $3.0-7.8$ & & & $\begin{array}{l}\text { horse1 (12), EbpA (1), } \\
\text { EbpC (2), Peru6 (3), } \\
\text { SXH-1 (1) }\end{array}$ \\
\hline $\begin{array}{l}\text { Castrated } \\
\text { horse }\end{array}$ & $2 / 85(2.4)$ & $0-5.6$ & & & horse1 (2) \\
\hline
\end{tabular}

their lower E. bieneusi infection rates compared with those of other horses.

In the present study, the prevalence rate significantly differed by age group: $16.2 \%(12 / 74)$ in young racehorses and 3.3\% $(18 / 547)$ in adults $\left(\chi^{2}=23.686, d f=1, p<0.01\right.$; Table 3). Similar findings were reported in a Colombian study, with infection rates of $23.7 \%(18 / 76)$ in young horses and $2.5 \%(3 / 119)$ in adult horses (Santín et al., 2010). However, previous reports from the Czechia and Algeria and from Xinjiang, Sichuan, and Yunnan in China, showed no statistical differences among the age groups of the horses (Wagnerová et al., 2012; Laatamna et al., 2015; Deng et al., 2016b; Qi et al., 2016). Furthermore, no significant differences were found in the prevalence rates among stallions $(4.8 \%, 9 / 186)$, mares $(5.4 \%, 19 / 350)$ and castrated horses $(2.4 \%$, $2 / 85)\left(\chi^{2}=1.407, d f=2, p>0.05\right.$; Table 3$)$. This result is similar to those of previous reports conducted in China, Colombia, Algeria and the Czechia (Santín et al., 2010; Wagnerová et al., 2012; Laatamna et al., 2015; Deng et al., 2016b; Qi et al., 2016). Limited data on this topic suggest that more studies are needed to determine the relationship between age, sex and E. bieneusi infections in horses.

In the present study, 10 E. bieneusi genotypes were identified in racehorses, including seven known genotypes (EbpC, EbpA, Peru6, horse1, horse2, CAF1, and TypeIV) and three novel genotypes (HBH-1, SXH-1, and $\mathrm{BJH}-1)$. The three novel genotypes, $\mathrm{HBH}-1, \mathrm{SXH}-1$, and $\mathrm{BJH}-1$, were closely related to genotypes BEB4, Peru6, and Henan-I, with one, two, and four single nucleotide polymorphisms, respectively. Genotype HBH1 had one nucleotide substitution $(\mathrm{T} \rightarrow \mathrm{C}$ ) relative to genotype BEB4. Genotype SXH-1 had two nucleotide substitutions $(\mathrm{G} \rightarrow \mathrm{A}$ and $\mathrm{C} \rightarrow \mathrm{T}$ ) relative to genotype Peru6. Genotype $\mathrm{BJH}-1$ had four nucleotide substitutions $(\mathrm{G} \rightarrow \mathrm{A}, \mathrm{G} \rightarrow \mathrm{A}, \mathrm{T} \rightarrow \mathrm{C}$, and $\mathrm{T} \rightarrow \mathrm{C}$ ) relative to genotype Henan-I. Among the ten genotypes, horse1 $(53.3 \%, 16 / 30)$ dominated, followed by Peru6 $(13.3 \%, 4 / 30)$, horse2 $(6.7 \%, 2 / 30), \operatorname{EbpC}(6.7 \%, 2 / 30)$, and each of the remaining 
six genotypes $(3.3 \%, 1 / 30)$. Our phylogenetic analysis revealed that the EbpC, EbpA, TypeIV, Peru6, CAF1, horse1, SXH-1, and BJH-1 genotypes clustered into Group 1, HBH-1 into Group 2, and horse2 into Group 6 (Figure 2). Among these genotypes, EbpA, EbpC, Peru6, and TypeIV have been identified in a wide range of hosts, including humans, non-human primates, pets,

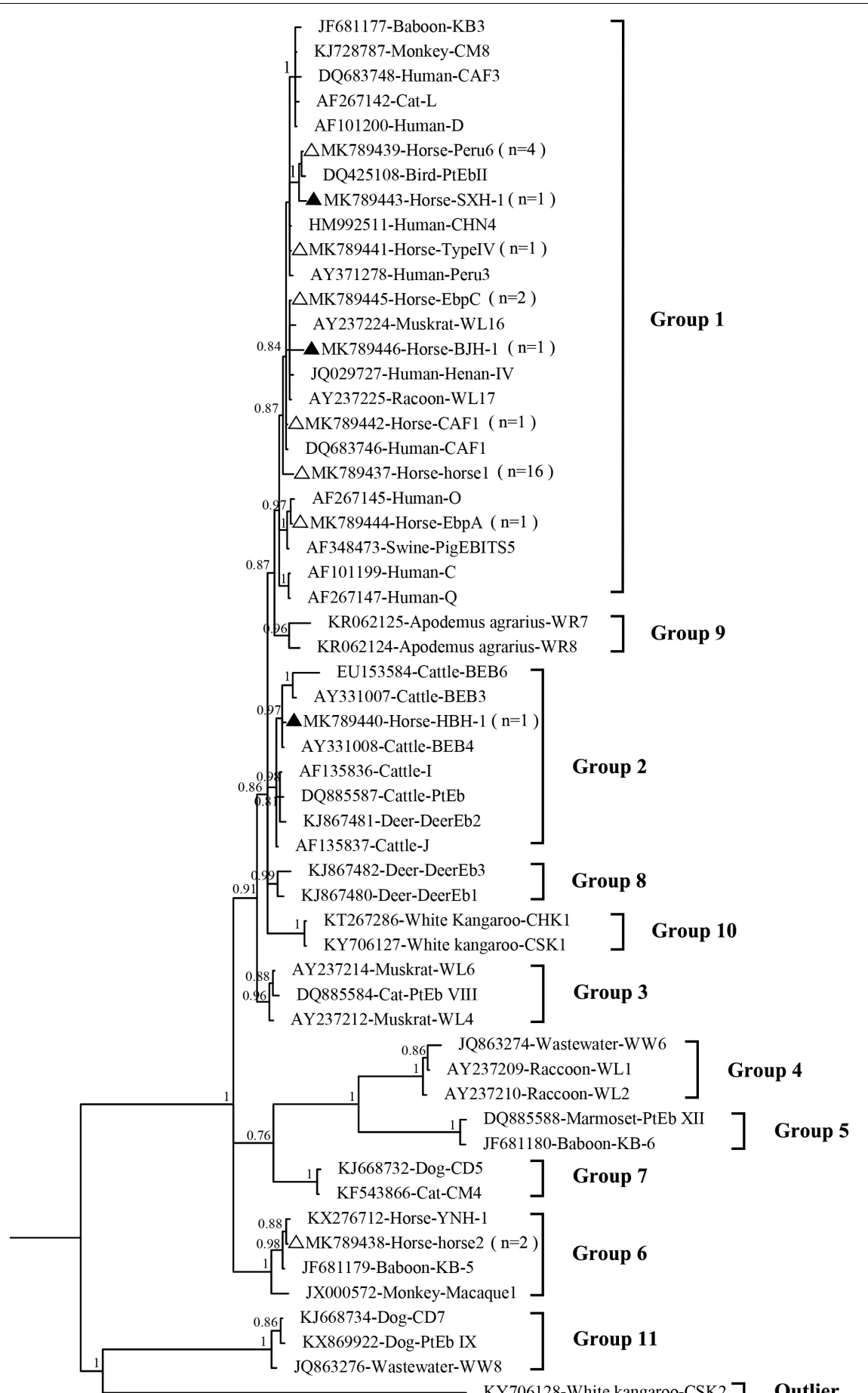

0.08

FIGURE 2 | Phylogenetic tree based on Bayesian analysis of the ITS sequences. Statistically significant posterior probabilities are indicated on the branches. The specimen names include the GenBank accession numbers followed by the host and genotype designation. The E. bieneusi genotype CSK2 (KY706128) from the white kangaroo was used as the outlier. Known and new genotypes are indicated by hollow and filled triangles, respectively. 
domestic animals and wild animals (Santín and Fayer, 2011; Wang et al., 2018). Genotype horsel has been found in horses and non-human primates, genotype horse 2 has been found in horses, black bears and squirrels, and genotype CAF1 has been found in horses, pigs, goats, deer and humans (Breton et al., 2007; Jeong et al., 2007; Wagnerová et al., 2012; Deng et al., 2016a, 2017; Qi et al., 2016; Shi et al., 2016; Zhong et al., 2017). Genotypes EbpC and Type IV have been detected in humans in several regions of China (Karim et al., 2014; Yang et al., 2014; Liu et al., 2017; Wang et al., 2017). Future studies should evaluate the molecular epidemiology of E. bieneusi genotypes in horses and other animals to better elucidate its transmission dynamics.

Possible genotypic differences in E. bieneusi dominance in horses in different geographic areas should also be determined. Dominant genotypes were observed, such as horse1 in Colombia, genotype $\mathrm{D}$ in the Czechia, horse 2 in Sichuan and Yunnan, China, and the dominant genotypes EbpA and EbpC in Xinjiang, China (Santín et al., 2010; Wagnerová et al., 2012; Laatamna et al., 2015; Deng et al., 2016b; Qi et al., 2016; Tables 2, 3). Therefore, E. bieneusi infections in horses likely differ regionally.

\section{CONCLUSION}

Our results revealed a relatively low occurrence of E. bieneusi in racehorses. Ten E. bieneusi genotypes were identified, with horse1 being predominant. The observations of five genotypes (EbpC, EbpA, Peru6, TypeIV, and CAF1) in humans as well as three novel genotypes (BGH-1 and SXH-1 in Group 1 and $\mathrm{HBH}$ 1 in Group 2) suggest the possibility that racehorses may transmit E. bieneusi to humans.

\section{DATA AVAILABILITY}

The datasets generated for this study can be found in the GenBank under the accession numbers MK789437-MK789446.

\section{REFERENCES}

Ben, A. L., Yang, W., Widmer, G., Cama, V., Ortega, Y., and Xiao, L. (2012). Survey and genetic characterization of wastewater in Tunisia for Cryptosporidium spp., Giardia duodenalis, Enterocytozoon bieneusi, Cyclospora cayetanensis and Eimeria spp. J. Water Health 10, 431-444. doi: 10.2166/wh.2012.204

Breitenmoser, A. C., Mathis, A., Bürgi, E., Weber, R., and Deplazes, P. (1999). High prevalence of Enterocytozoon bieneusi in swine with four genotypes that differ from those identified in humans. Parasitology 118(Pt 5), 447-453. doi: $10.1017 /$ S0031182099004229

Breton, J., Bart-Delabesse, E., Biligui, S., Carbone, A., Seiller, X., Okome-Nkoumou, M., et al. (2007). New highly divergent rRNA sequence among biodiverse genotypes of Enterocytozoon bieneusi strains isolated from humans in Gabon and Cameroon. J. Clin. Microbiol. 45, 2580-2589. doi: 10.1128/JCM.02 554-06

Buckholt, M. A., Lee, J. H., and Tzipori, S. (2002). Prevalence of Enterocytozoon bieneusi in swine: an 18-month survey at a slaughterhouse in Massachusetts. Appl. Environ. Microbiol. 68, 2595-2599. doi: 10.1128/AEM.68.5.2595-2599. 2002

Decraene, V., Lebbad, M., Botero-Kleiven, S., Gustavsson, A. M., and Löfdahl, M. (2012). First reported foodborne outbreak associated with microsporidia, Sweden, October 2009. Epidemiol. Infect. 140, 519-527. doi: 10.1017/S09502688 $1100077 \mathrm{X}$

\section{ETHICS STATEMENT}

The Ethics Review Committee of Henan Agricultural University reviewed and approved this research under the approval number IRC-HENAU-20160225. The equestrian club owners granted permission for specimen collection. Animals were handled in accordance with the Animal Ethics Procedures and Guidelines of the People's Republic of China.

\section{AUTHOR CONTRIBUTIONS}

MQ and LZ designed the study. AZ, ZW, YP, and YnZ collected and analyzed the specimens. DL and YxZ analyzed the data. AZ, MQ, and LZ wrote the manuscript. All authors read and approved the final manuscript.

\section{FUNDING}

This work was supported in part by the National Natural Science Foundation of China (31860699 and 31660712) and the Program for Young and Middle-aged Leading Science, Technology, and Innovation of Xinjiang Production \& Construction Group (2018CB034). The sponsors played no roles in the study design or in the collection, analysis, or interpretation of the data, in writing the report, or in the decision to submit the article for publication.

\section{ACKNOWLEDGMENTS}

We thank Traci Raley, MS, ELS, from Liwen Bianji, Edanz Editing China (www.liwenbianji.cn/ac), for editing a draft of this manuscript.

Deng, L., Li, W., Zhong, Z., Gong, C., Cao, X., Song, Y., et al. (2017). Multi-locus genotypes of Enterocytozoon bieneusi in captive Asiatic black bears in southwestern China: high genetic diversity, broad host range, and zoonotic potential. PLoS One 12:e0171772. doi: 10.1371/journal.pone.017 1772

Deng, L., Li, W., Yu, X., Gong, C., Liu, X., Zhong, Z., et al. (2016a). First report of the human-pathogenic Enterocytozoon bieneusi from red-bellied tree squirrels (Callosciurus erythraeus) in Sichuan, China. PLoS One 11:e0163605. doi: 10. 1371/journal.pone.0163605

Deng, L., Li, W., Zhong, Z., Gong, C., Liu, X., Huang, X., et al. (2016b). Molecular characterization and multilocus genotypes of Enterocytozoon bieneusi among horses in southwestern China. Parasit. Vectors. 9:561. doi: 10.1186/s13071-0161844-3

Didier, E. S., and Weiss, L. M. (2006). Microsporidiosis: current status. Curr. Opin. Infect. Dis. 19, 485-492. doi: 10.1097/01.qco.0000244055.463 82.23

Didier, E. S., Weiss, L. M., Cai, A., and Marciano-Cabral, F. (2009). Overview of the presentations on microsporidia and free-living amebae at the 10th international workshops on opportunistic protists. Eukaryot. Cell. 8, 441-445. doi: 10.1128/ EC.00302-08

Galván, A. L., Magnet, A., Izquierdo, F., Fenoy, S., Rueda, C., Fernández Vadillo, C., et al. (2013). Molecular characterization of human-pathogenic microsporidia and Cyclospora cayetanensis isolated from various water sources 
in Spain: a year-long longitudinal study. Appl. Environ. Microbiol. 79, 449-459. doi: 10.1128/AEM.02737-12

Jeong, D. K., Won, G. Y., Park, B. K., Hur, J., You, J. Y., Kang, S. J., et al. (2007). Occurrence and genotypic characteristics of Enterocytozoon bieneusi in pigs with diarrhea. Parasitol. Res. 102, 123-128. doi: 10.1007/s00436-0070740-3

Karim, M. R., Wang, R., He, X., Zhang, L., Li, J., Rume, F. I., et al. (2014). Multilocus sequence typing of Enterocytozoon bieneusi in nonhuman primates in China. Vet. Parasitol. 200, 13-23. doi: 10.1016/j.vetpar.2013.12.004

Laatamna, A. E., Wagnerová, P., Sak, B., Kvìtoòová, D., Xiao, L., Rost, M., et al. (2015). Microsporidia and Cryptosporidium in horses and donkeys in Algeria: detection of a novel Cryptosporidium hominis subtype family (Ik) in a horse. Vet. Parasitol. 208, 135-142. doi: 10.1016/j.vetpar.2015.01.007

Leelayoova, S., Subrungruang, I., Suputtamongkol, Y., Worapong, J., Petmitr, P. C., and Mungthin, M. (2006). Identification of genotypes of Enterocytozoon bieneusi from stool samples from human immunodeficiency virus-infected patients in Thailand. J. Clin. Microbiol. 44, 3001-3004. doi: 10.1128/JCM.009 45-06

Li, N., Xiao, L., Wang, L., Zhao, S., Zhao, X., Duan, L., et al. (2012). Molecular surveillance of Cryptosporidium spp., Giardia duodenalis, and Enterocytozoon bieneusi by genotyping and subtyping parasites in wastewater. PLoS Negl. Trop. Dis. 6:e1809. doi: 10.1371/journal.pntd.0001809

Li, W., Feng, Y., and Santin, M. (2019). Host specificity of Enterocytozoon bieneusi and public health implication. Trends Parasitol. 35, 436-451. doi: 10.1016/j.pt. 2019.04.004

Li, W., and Xiao, L. (2019). Multilocus sequence typing and population genetic analysis of Enterocytozoon bieneusi: host specificity and its impacts on public health. Front. Genet. 10:307. doi: 10.3389/fgene.2019.00307

Liu, H., Jiang, Z., Yuan, Z., Yin, J., Wang, Z., Yu, B., et al. (2017). Infection by and genotype characteristics of Enterocytozoon bieneusi in HIV/AIDS patients from Guangxi Zhuang autonomous region, China. BMC Infect. Dis. 17:684. doi: 10.1186/s12879-017-2787-9

Lores, B., del Aguila, C., and Arias, C. (2002). Enterocytozoon bieneusi (microsporidia) in faecal samples from domestic animals from Galicia, Spain Mem. Inst. Oswaldo. Cruz. 97, 941-945. doi: 10.1590/S0074-027620020007 00003

Maikai, B. V., Umoh, J. U., Lawal, I. A., Kudi, A. C., Ejembi, C. L., and Xiao, L. (2012). Molecular characterizations of Cryptosporidium, Giardia, and Enterocytozoon in humans in Kaduna State, Nigeria. Exp. Parasitol. 131, 452456. doi: 10.1016/j.exppara.2012.05.011

Qi, M., Wang, R., Wang, H., Jian, F., Li, J., Zhao, J., et al. (2016). Enterocytozoon bieneusi genotypes in grazing horses in China and their zoonotic transmission potential. J. Eukaryot. Microbiol. 63, 591-597. doi: 10.1111/jeu.12308

Sak, B., Brady, D., Pelikánová, M., Kvitoòová, D., Rost, M., Kostka, M., et al. (2011). Unapparent microsporidial infection among immunocompetent humans in the Czech Republic. J. Clin. Microbiol. 49, 1064-1070. doi: 10.1128/JCM.01147-10

Santín, M., Calero-Bernal, R., Carmena, D., Mateo, M., Balseiro, A., Barral, M., et al. (2018). Molecular characterization of Enterocytozoon bieneusi in wild carnivores in Spain. J. Eukaryot. Microbiol. 65, 468-474. doi: 10.1111/jeu. 12492

Santín, M., and Fayer, R. (2011). Microsporidiosis: Enterocytozoon bieneusi in domesticated and wild animals. Res. Vet. Sci. 90, 363-371. doi: 10.1016/j.rvsc. 2010.07.014
Santín, M., Vecino, J. A., and Fayer, R. (2010). A zoonotic genotype of Enterocytozoon bieneusi in horses. J. Parasitol. 96, 157-161. doi: 10.1645/GE2184.1

Shi, K., Li, M., Wang, X., Li, J., Karim, M. R., Wang, R., et al. (2016). Molecular survey of Enterocytozoon bieneusi in sheep and goats in China. Parasit. Vectors 9:23. doi: 10.1186/s13071-016-1304-0

Thellier, M., and Breton, J. (2008). Enterocytozoon bieneusi in human and animals, focus on laboratory identification and molecular epidemiology. Parasite 15, 349-358. doi: 10.1051/parasite/2008153349

Wagnerová, P., Sak, B., Kvitoòová, D., Buòatová, Z., Civišová, H., Maršálek, M., et al. (2012). Enterocytozoon bieneusi and Encephalitozoon cuniculi in horses kept under different management systems in the Czech Republic. Vet. Parasitol. 190, 573-577. doi: 10.1016/j.vetpar.2012.07.013

Wagnerová, P., Sak, B., McEvoy, J., Rost, M., Sherwood, D., Holcomb, K., et al. (2016). Cryptosporidium parvum and Enterocytozoon bieneusi in American Mustangs and Chincoteague ponies. Exp. Parasitol. 162, 24-27. doi: 10.1016/ j.exppara.2015.12.004

Wang, L., Zhang, H., Zhao, X., Zhang, L., Zhang, G., Guo, M., et al. (2013). Zoonotic Cryptosporidium species and Enterocytozoon bieneusi genotypes in HIV-positive patients on antiretroviral therapy. J. Clin. Microbiol. 51, 557-563. doi: 10.1128/JCM.02758-12

Wang, S., Wang, R., Fan, X., Liu, T., Zhang, L., and Zhao, G. (2018). Prevalence and genotypes of Enterocytozoon bieneusi in China. Acta Trop. 183, 142-152. doi: 10.1016/j.actatropica.2018.04.017

Wang, T., Fan, Y., Koehler, A. V., Ma, G., Li, T., Hu, M., et al. (2017). First survey of Cryptosporidium, Giardia and Enterocytozoon in diarrhoeic children from Wuhan, China. Infect. Genet. Evol. 51, 127-131. doi: 10.1016/j.meegid.2017. 03.006

Yang, J., Song, M., Wan, Q., Li, Y., Lu, Y., Jiang, Y., et al. (2014). Enterocytozoon bieneusi genotypes in children in Northeast China and assessment of risk of zoonotic transmission. J. Clin. Microbiol. 52, 4363-4367. doi: 10.1128/JCM. 02295-14

Zhang, Y., Koehler, A. V., Wang, T., Haydon, S. R., and Gasser, R. B. (2018). First detection and genetic characterisation of Enterocytozoon bieneusi in wild deer in Melbourne's water catchments in Australia. Parasit. Vectors 11:2. doi: 10.1186/s13071-017-2577-7

Zhong, Z., Li, W., Deng, L., Song, Y., Wu, K., Tian, Y., et al. (2017). Multilocus genotyping of Enterocytozoon bieneusi derived from nonhuman primates in southwest China. PLoS One 12:e0176926. doi: 10.1371/journal.pone.0176926

Conflict of Interest Statement: YP was employed by the company Equivets, China.

The remaining authors declare that the research was conducted in the absence of any commercial or financial relationships that could be construed as a potential conflict of interest.

Copyright (C) 2019 Zhao, Li, Wei, Zhang, Peng, Zhu, Qi and Zhang. This is an open-access article distributed under the terms of the Creative Commons Attribution License (CC BY). The use, distribution or reproduction in other forums is permitted, provided the original author(s) and the copyright owner(s) are credited and that the original publication in this journal is cited, in accordance with accepted academic practice. No use, distribution or reproduction is permitted which does not comply with these terms. 